\title{
BMJ Open Qualitative descriptive study to explore nurses' perceptions and experience on pain, agitation and delirium management in a community intensive care unit
}

\author{
Jennifer L Y Tsang, ${ }^{\oplus 1,2}$ Katie Ross, ${ }^{2}$ Franziska Miller, ${ }^{3}$ Ramez Maximous, ${ }^{2}$ \\ Priscilla Yung, ${ }^{3}$ Carl Marshall, ${ }^{3}$ Mercedes Camargo, ${ }^{2}$ Dimitra Fleming, ${ }^{2}$ \\ Madelyn Law ${ }^{4}$
}

To cite: Tsang JLY, Ross $K$, Miller F, et al. Qualitative descriptive study to explore nurses' perceptions and experience on pain, agitation and delirium management in a community intensive care unit. BMJ Open 2019;9:e024328. doi:10.1136/ bmjopen-2018-024328

- Prepublication history for this paper is available online. To view these files, please visit the journal online (http://dx.doi. org/10.1136/bmjopen-2018024328).

Received 22 May 2018 Revised 16 January 2019 Accepted 12 February 2019

\section{Check for updates}

(C) Author(s) (or their employer(s)) 2019. Re-use permitted under CC BY-NC. No commercial re-use. See rights and permissions. Published by BMJ.

${ }^{1}$ Department of Medicine, McMaster University, Hamilton, Ontario, Canada

${ }^{2}$ Medicine, Niagara Health System-Saint Catharines Site, Saint Catharines, Ontario, Canada

${ }^{3}$ McMaster University Michael G DeGroote School of Medicine, Hamilton, Ontario, Canada

${ }^{4}$ Community Health Sciences, Brock University, St. Catherines, Ontario, Canada

Correspondence to Jennifer L Y Tsang; jennifer.tsang@mail.utoronto.ca

\section{ABSTRACT}

Objectives The purpose of this study was to explore the experiences, beliefs and perceptions of intensive care unit (ICU) nurses on the management of pain, agitation and delirium (PAD) in critically ill patients.

Design A qualitative descriptive study.

Setting This study took place in a community hospital ICU located in a medium size Canadian city.

Participants Purposeful sampling was conducted. Participants included full-time nurses working in the ICU. Forty-six ICU nurses participated.

Methods A total of five focus group sessions were held to collect data. There were one to three separate groups in each focus group session, with no more than seven participants in each group. There were 10 separate groups in total. A semistructured question guide was used. Thematic analysis method was adopted to analyse the data, and to search for emergent themes and patterns. Results Three main themes emerged: (1) the professional perspectives on patient wakefulness state, (2) the professional perspectives on PAD management of critically ill patients and (3) the factors impacting PAD management. Nurses have different opinions on the optimal level of patient sedation and felt that many factors, including environmental, healthcare teams, patients and family members, can influence PAD management. This potentially leads to inconsistent PAD management in critically ill patients. The nurses also believed that PAD management requires a multidisciplinary approach including healthcare teams and patients' families.

Conclusions Many external and internal factors contribute to the complexity of PAD management including the attitudes of nursing staff towards PAD. The themes emerged from this study suggested the need of a multifaceted and multidisciplinary quality improvement programme to optimise the management of PAD in the ICU.

\section{INTRODUCTION}

A majority of critically ill patients experience pain and agitation during their intensive care unit (ICU) stay. The lack of recognition
Strengths and limitations of this study

- This study examines the attitudes of nurses towards pain, agitation and delirium (PAD) management in a Canadian community intensive care unit (ICU).

- A total of six analysts from a diverse background performed data analysis (three generated codes and three developed themes) to allow researcher triangulation and to ensure validity of results.

- The use of focus group as a method of data collection to facilitate the emergence of personal and group beliefs, perceptions and opinions.

- There was no audio recording for transcription. However, there were dedicated notetakers to transcribe detailed notes including direct quotes to allow for data analysis.

- This is a single-centre study which might affect generalisability. However, this study was conducted in a community ICU rather than an academic ICU where most studies are conducted. Therefore, the results of this study contribute to new knowledge in the field of PAD research.

and improper treatment may contribute to the development of delirium, which is a common manifestation of acute brain dysfunction. ${ }^{1}$ The presence of delirium is associated with multiple complications and adverse outcomes, including prolonged hospital length of stay, ${ }^{2-6}$ increased healthcare costs, ${ }^{457}$ increased risk of postdischarge institutionalisation, ${ }^{8} \quad$ long-term cognitive dysfunction $^{910}$ and increased mortality both in hospital and after discharge. ${ }^{411}$ As a result, the Society of Critical Care Medicine developed professional guidelines in $2013^{12}$ for the management of pain, agitation and delirium (PAD) in adult patients in the ICU.

However, guideline implementation rates in the assessment and treatment of PAD are 
suboptimal. ${ }^{13-16}$ Barriers to guideline implementation include organisational, professional and personal factors such as lack of training, skills, knowledge, motivation and culture of change. ${ }^{17-24}$ The Consolidated Framework for Implementation Research has described the importance of individuals' knowledge and beliefs about the intervention on the success of implementation. ${ }^{25}{ }^{26}$ Specifically, the individuals' attitudes towards and value placed on the interventions, as well as familiarity with the facts, truths and principles related to the interventions are crucial to guideline implementation. ${ }^{25}$ Devlin et al suggested some potential barriers to PAD guidelines implementation. In his study, he showed that only $3 \%$ of ICU nurses ranked delirium as the most important condition to evaluate, compared with level of consciousness (44\%), presence of pain $(23 \%)$ or improper placement of an invasive device $(21 \%) .{ }^{27}$ Moreover, ICU nurses reported many barriers to delirium assessment including intubation (38\%), the complexity of tools for assessing delirium $(34 \%)$ and the inability to complete assessments of delirium in sedated patients $(13 \%){ }^{27}$

In a qualitative study, Palacios-Ceña et al explored the experiences of physicians and nurses caring for patients with delirium in the ICU in Spain. They found that healthcare professionals felt that delirium is a complex but non-urgent condition, therefore resulting in variable management strategies. ${ }^{28}$ This suggests the need for change in healthcare professionals' attitudes towards delirium management in order to overcome the barriers to PAD guidelines implementation.

The Promoting Action on Research Implementation in Health Services Framework suggests that the process of guideline implementation plays a significant role in implementation success. ${ }^{26}$ The 2013 PAD guidelines recommend regular assessment of PAD using validated tools, prompt treatment of PAD guided by validated tools and prevention of PAD by nurses. ${ }^{12}$ To facilitate the implementation of the PAD guidelines by targeting the potential barriers to implementation at the professional and personal level of nurses, we conducted a qualitative descriptive study using focus groups to explore ICU nurses' experiences, beliefs and perceptions on PAD management in a Canadian community ICU. The results of this study will guide the development of quality improvement interventions with the aim of improving $\mathrm{PAD}$ management and decreasing the rate of delirium in community ICUs.

\section{METHODS}

We followed the Standards for Reporting Qualitative Research: A Synthesis of Recommendations during the reporting of this study. ${ }^{29}$

\section{Study design}

This study adopted a qualitative descriptive methodology that provides a rich, and thick description ${ }^{30}$ of the participants' perceptions that stays close to the data. ${ }^{31}$ Focus groups were used as a method of data collection. Focus groups are small groups of individuals who share common characteristics or experiences. ${ }^{32}$ They allow researchers to observe group dynamics and to explore what people think and how and why they think as they do. ${ }^{33}$

\section{Objectives}

The purpose of this study was to explore the experiences, beliefs and perceptions of ICU nurses on the management of PAD in critically ill patients.

Our two main research questions are as follows:

1. What are nurses' perceptions and experience with PAD management in the ICU?

2. What are the barriers to evidence-based PAD management practices in the ICU?

\section{Setting}

This study took place in a community hospital located in a medium size Canadian city, where more than 90 parttime and full-time registered nurses provide ICU care. This centre has one adult 14-bed (all individual rooms) level-III medical-surgical ICU (where the intensivist acts as the primary physician during the patients' ICU stay), with a nurse to patient ratio of 1:1 to 1:2. There is a dedicated ICU pharmacist, respiratory therapist, physiotherapist, dietitian and 24 hours in-house intensivist coverage. The ICU patient population includes general medical, cardiac, respiratory, nephrology, oncology, general surgical, orthopaedic and vascular surgical patients. The ICU is a locked unit but without restriction in family visiting hours. There is a nursing policy in place to stipulate that pain is assessed using the Numeric Pain Rating Scale at the beginning of each shift, after analgesic administration every 4 hours, and as needed. Sedation level is assessed using the Richmond Agitation Sedation Scale at the beginning of each shift and as needed and delirium is assessed using the Confusion Assessment for the ICU. The ICU nurses are responsible for titrating doses and frequency of analgesics and sedatives according to patients' clinical status. There is no pre-existing protocol for medication titration.

\section{Research team and reflexivity}

A total of 10 researchers participated in this study. KR was a research assistant with a Masters' degree in health sciences and experience in qualitative research. RM was a research volunteer with an undergraduate degree in health sciences. FM, PY and CM were medical students. MC was a research volunteer with a medical degree from Colombia. DF was a pharmacist in the ICU where the study was conducted. ML was a PhD scientist with qualitative research expertise. JLYT was an $\mathrm{MD} / \mathrm{PhD}$ clinician scientist and intensivist in the ICU where the study was conducted. KR, RM, FM, PY, CM, MC and ML had no clinical involvement in the ICU and no relationship with study participants. Both DF and JLYT worked in the ICU where the study was conducted and had relationships with study participants. 
During study design, the positioning of JLYT and ML, first author and senior author, respectively, was established regarding the researchers' belief (suboptimal PAD management in the ICU) and motivation for the research (to improve PAD management in the ICU). Researchers based their approach on a constructivist paradigm with an assumption that knowledge is constructed based on personal experiences and hypotheses of environment. ${ }^{3435}$

\section{Participants and sampling}

Purposeful sampling was conducted to select information-rich cases for study in depth. Information-rich cases are those from whom one can learn a great deal about issues of central importance to the purpose of the research. ${ }^{36}$ Moreover, the sampling for focus groups typically involves bringing together people of similar backgrounds and experiences. Therefore, purposeful sampling strategy was used. ${ }^{37}$ The inclusion criteria consisted of nurses who worked at full-time capacity in the community ICU (single ICU). There were no exclusion criteria.

An email was sent to invite nurses to participate in the study. A sign-up sheet was posted in the ICU where nurses who wished to participate could place their names. A few days before the focus group sessions, a reminder email was sent to participants. Participants were reimbursed on an hourly basis for their time spent in the focus group session. Of the 76 full-time ICU nurses who were eligible and invited, a total of 66 signed up and 46 participated. These included 43 women and three men. The gender ratio is representative of the nursing demographics in the ICU. There were 16 no shows.

\section{Ethical considerations}

We ensured participant confidentiality by anonymising our data. The participants were all informed of the purpose of the study and the voluntary nature of their participation.

\section{Data collection}

Semistructured focus group sessions were conducted in a hospital meeting room away from the ICU where participants worked. This data collection strategy was chosen to allow participants to discuss, explore, describe, explain, share and compare their thoughts, ideas and perceptions. ${ }^{30}$ There were five focus group session times that participants could choose from. At each session, there were 1-3 focus groups occurring with group sizes ranging from 3 to 7 participants (table 1). For each focus group, there was an experienced facilitator and a dedicated notetaker (FM, CM and PY) to take extensive notes in real time during the focus groups using a laptop. The notes include direct quotes from participants.

The facilitators (JLYT, DF and ML) conducted the focus groups with a semistructured interview guide with six open-ended questions (table 2) to guide discussions in English. The interview guide questions were informed by the responses to a nurse survey conducted prior to the design of this study ${ }^{38}$ together with sensitising concepts from existing literature. They were formulated to address the participants' perceptions, beliefs and experience in PAD management and their perceived barriers to optimal PAD management in the ICU. The facilitators first explained the purposes of the focus groups and asked all participants to sign an informed consent form. The facilitators, then followed the semi-structured interview guide by posing one question at a time, allowing time

Table 1 Characteristics of the study participants and focus groups (FGs)

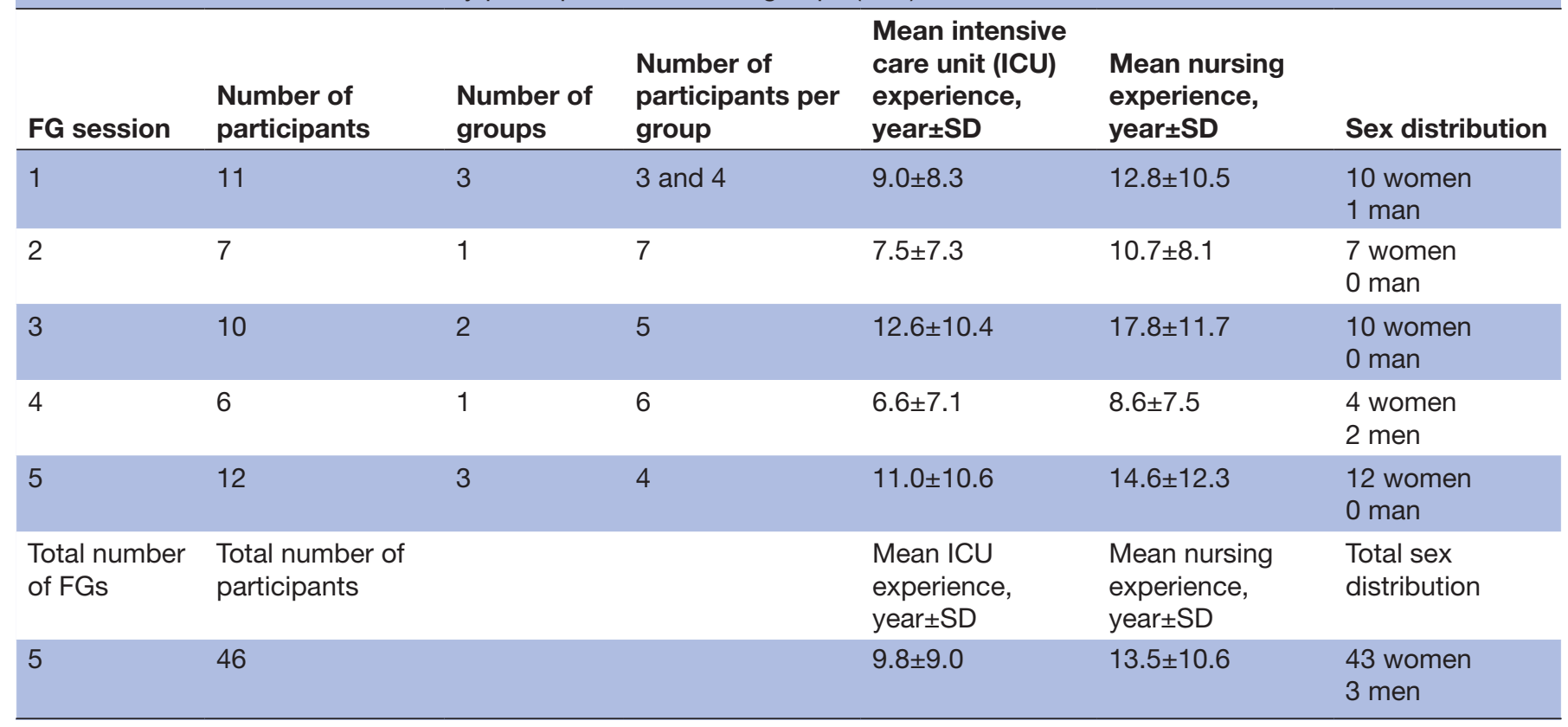




\begin{tabular}{|c|c|c|}
\hline Phase & Contents & Time (min) \\
\hline Opening & $\begin{array}{l}\text { Facilitator welcome the } \\
\text { participants and explained the } \\
\text { purpose of the focus group and } \\
\text { asked participants to sign an } \\
\text { informed consent form }\end{array}$ & $5-7$ \\
\hline Question 1 & $\begin{array}{l}\text { What would you say an ideal } \\
\text { intensive care unit (ICU) } \\
\text { ventilated patient should look } \\
\text { like? Why? }\end{array}$ & 50 \\
\hline Question 2 & $\begin{array}{l}\text { What does a problematic ICU } \\
\text { ventilated patient look like from } \\
\text { your perspective as a healthcare } \\
\text { provider? }\end{array}$ & \\
\hline Question 3 & $\begin{array}{l}\text { If you were an ICU ventilated } \\
\text { patient, how would you want to } \\
\text { be sedated? Why? }\end{array}$ & \\
\hline Question 4 & $\begin{array}{l}\text { How do we contribute to the } \\
\text { development of delirium in the } \\
\text { ICU? }\end{array}$ & \\
\hline Question 5 & $\begin{array}{l}\text { How can we help you better } \\
\text { manage pain, agitation and } \\
\text { delirium (PAD) in the ICU? What } \\
\text { are the challenges in the ICU that } \\
\text { make it difficult to manage PAD? }\end{array}$ & \\
\hline Question 6 & $\begin{array}{l}\text { How can we better engage you in } \\
\text { changes to improve care for our } \\
\text { patients? }\end{array}$ & \\
\hline Closing & $\begin{array}{l}\text { Facilitator thanked the } \\
\text { participants for participating in } \\
\text { the focus group. }\end{array}$ & 3 \\
\hline
\end{tabular}

for participants to voice their opinions and for discussion among the group. After all questions were posed, facilitators ended the focus groups with closing remarks (table 2). Since there was no audio recording, we did not have the exact duration of the focus groups. However, all focus groups lasted approximately $60 \mathrm{~min}$. All the research data were anonymised and kept confidential.

\section{Data analysis}

Thematic analysis was adopted to search for themes and patterns in the focus group data. ${ }^{31}$ Initially, three independent analysts (FM, RM and CM) read the data sets multiple times to familiarise themselves with the data to generate and assign initial codes to units of meaning. Open coding (fracturing of the data and grouping/categorising) was performed, followed by axial coding (rearranging the data in new ways). All three of the analysts who generated and assigned initial codes (FM, RM and CM) did not have any clinical roles in the ICU; therefore, they did not have preconceived biases in regard to the quality of PAD management in the ICU. Moreover, one of the analysts (RM) had no prior involvement in the design and conduct of this study. This allowed RM to conduct the analysis with tabula rasa (blank slate). A total of 10 focus

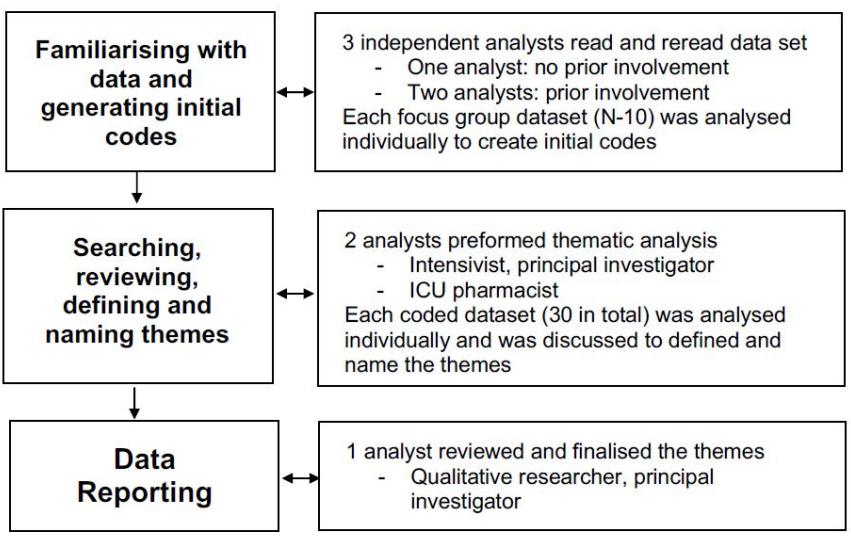

Figure 1 Data analysis scheme. ICU, intensive care unit.

group data sets were analysed separately to create initial codes. Then two analysts performed thematic analysis by searching, reviewing, defining and naming themes. One analyst (JLYT) was an intensivist who is the first author of this manuscript and the other analyst (DF) was an ICU pharmacist. A total of 30 coded data sets were analysed individually and then discussed as a group to develop, define and name the themes. Finally, one analyst (ML), who is a qualitative researcher and the senior author of this study reviewed and finalised the themes (see figure 1). No qualitative software was used on the data.

\section{Trustworthiness}

The trustworthiness of this study was ensured by enhancing its credibility, transferability and dependability. ${ }^{39}$ Credibility was achieved using (1) a well-established research methodology (qualitative descriptive) and method (focus group), (2) triangulation as the focus of the interview questions was informed by results of a previous survey conducted by our group ${ }^{38}$ and (3) interview questions that were designed to collect descriptive data that reflected real experiences and perspectives of participants. Finally, despite the lack of audio recording, three transcribers were responsible for obtaining detailed transcripts including direct quotes in a total of 10 separate data sets. The credibility of the data sets was ensured by the similarity of the codes and themes that were generated and developed from these 10 separate data sets.

We addressed the transferability of this study by providing a detailed description of the setting (community ICU in a medium size Canadian city) and context (the aim of this study was to inform the development of PAD quality improvement interventions) in which this study took place. Further, reflexive insight from the researchers was noted, with the motives of researchers being to understand and improve PAD management, and the perception that PAD management in its current state was suboptimal within the community hospital setting. The dependability of this study was upheld by performing audit trails throughout the process of collecting, coding and thematising the data. We also performed member checking by asking four participants to review the results 
of our study. They felt that the results were consistent with their beliefs, perceptions and experiences. Our member checking validated the results of our study. Finally, we performed researcher triangulation by using multiple analysts to review data sets, generate codes and develop themes to reduce bias.

\section{Patient and public involvement statement}

The patients and the public were not involved in the design or planning of the study.

\section{RESULTS}

A total of 10 focus groups with a total of 46 participants were conducted resulted in 10 separate data sets. By using thematic analysis, three main themes emerged from the data: (1) the professional perspectives on patient wakefulness state, (2) the professional perspectives on PAD management of critically ill patients and (3) the factors impacting PAD management (table 3).

\section{Theme: the professional perspectives on patient wakefulness} state

This theme describes the divergent perspectives of nurses on optimal patient wakefulness state. Participants had polarised opinions on the appropriate wakefulness level of critically ill patients. The difference in perspectives appeared to stem from personal choice when thinking about how they would like to be treated as a patient.

\section{Subtheme: when to worry?}

Participants described divergent opinions on what is considered a harmful patient wakefulness state. Some reported that a patient who is deeply sedated would be

Table 3 Themes and representative quotations from study participants

Themes Representative quotations

\begin{tabular}{|c|c|c|}
\hline $\begin{array}{l}\text { The professional } \\
\text { perspectives on patient } \\
\text { wakefulness state }\end{array}$ & When to worry? & $\begin{array}{l}\text { 'If a patient is agitated, we can fix it.' (FG3) } \\
\text { 'If the patient is comatose, we are more worried, you may not be able to do } \\
\text { anything to help them.' (FG3) } \\
\text { 'Agitated patients are more call to action, annoying and keeping you busy.' (FG2) } \\
\text { 'Trying to get out of bed when they are not supposed to.' (FG2) }\end{array}$ \\
\hline & $\begin{array}{l}\text { The optimal patient } \\
\text { wakefulness state }\end{array}$ & $\begin{array}{l}\text { 'I don't want to remember anything because of fear of PTSD.' (FG5) } \\
\text { 'Better when they are able to communicate with nurses and follow instructions.' } \\
\text { (FG2) } \\
\text { 'I want to be able to know everything about the current situation and to make } \\
\text { decisions on healthcare management.' (FG4) }\end{array}$ \\
\hline
\end{tabular}

$\begin{array}{lll}\begin{array}{l}\text { The professional } \\ \text { perspectives on pain, }\end{array} & \begin{array}{l}\text { Clinical conditions of } \\ \text { patients }\end{array} & \begin{array}{l}\text { 'Patient coming in with previous dementia or Alzheimer makes delirium } \\ \text { management difficult.' (FG3) }\end{array} \\ \begin{array}{l}\text { (PAD) management of } \\ \text { 'Fentanyl is good for pain unless patient has already developed tolerance.' (FG1) }\end{array} & \text { 'Propofol is great if not hemodynamically compromised.' (FG1) }\end{array}$
critically ill patients

Preferences of healthcare providers

$\begin{array}{ll}\text { The factors impacting } & \begin{array}{l}\text { Interprofessional } \\ \text { dynamics }\end{array}\end{array}$

\section{Environmental factors}

Family Input
'Some doctors take a more conservative treatment while others use multiple drugs.' (FG2)

'Night nurses heavily sedate patients for easier management-takes longer for day staff to assess patients since it takes time to wean patient off sedation.' (FG2)

'The nurses like to work together, like a team, which will make a difference, but it depends on the staff.' (FG1)

'Nurses don't explain to patients what they are doing.' (FG3)

'Talk to patient like they are, they can hear us, be gentle.' (FG3)

'Patient should be told what's going on.' (FG3)

'Don't approach patient too abruptly.' (FG4)

'Overhead announcements and monitors with constant beeping are distracting and preventing good quality of sleep.' (FG1)

'Very bright light-difficult to regulate sleep patterns. ' (FG2)

'Loss of day night cycle if the patient is not facing the window.' (FG1)

'Line insertions, intubations, chest tube insertion, X-ray, blood work at 6 am disrupt sleep.' (FG2)

'Do not want family splitting the team or pitting nurses against each other.' (FG2) 'Family induces stress in patient, agitating patient, talking at patient, and not allowing patient to rest. ' (FG3)

'Educational material available to families might eliminate inconsistent practices such as different nurses telling patient families different things.' (FG3)

'Educational pamphlets to family to teach them how to manage from nonpharmacological side would help.' (FG5)

'It would be helpful if the family knew what patients' wishes are, whether they want to be completely sedated or conscious/aware.' (FG2) 
very worrisome because it precludes proper assessment of neurological function. On the other hand, some participants reported that a patient who is awake and agitated could cause self-harm if the patient was 'bucking the vent', 'pulling out lines and tubes' or 'banging, climbing in bed, [or] trying to extubate themselves.' It was also perceived by the participants that agitated patients could be harmful to healthcare workers through violent outbursts. Therefore, an agitated patient would be more concerning than a deeply sedated patient.

Moreover, the divergent opinions also stemmed from the perspectives of patient care. Specifically, some participants felt that it is much easier to provide nursing care to a deeply sedated patient compared with an awake and agitated patient. Others felt that an awake and agitated patient is much more demanding of constant nursing attention, precluding the nurses from attending to other patient care matters.

\section{Subtheme: the optimal patient wakefulness state}

Participants expressed polarised views on what is considered an optimal patient wakefulness state. Some participants were adamant that a critically ill patient should be deeply sedated. This view stemmed from both professional beliefs and personal preferences. Professionally, some participants strongly believed that an ICU experience would lead to post-traumatic stress disorder (PTSD) in patients who are kept awake. Therefore, they were adamant that critically ill patients should be deeply sedated. Moreover, some participants clearly voiced their demand of being deeply sedated should they become a critically ill patient. Their rationale primarily surrounded the risk of PTSD development from an ICU stay.

On the other hand, some participants felt that patients should be kept calm and awake. They believed that the most important thing is to manage the patients' pain. Once that is achieved, patients could be comfortably kept more awake. Some participants stated that they would prefer to be more awake should they become critically ill because they would like to be able to participate in medical decision making as a patient.

\section{Theme: the professional perspectives on PAD management of critically ill patients}

The second main theme that emerged from our data related to the professional perspectives on PAD management that were dependent on both patient status and healthcare providers' preferences, resulting in significant variability. This variability occasionally became the source of healthcare providers' frustrations.

\section{Clinical conditions of patients}

Participants reported that PAD management should be tailored to individual patients. The patient factors that need to be considered include severity of illness, underlying comorbidities such as cognitive impairment, drug metabolism (response to analgesics, sedatives and antipsychotics) and preferences. The participants highlighted that all patients respond differently to various medications and environmental stimuli. They also reported that patients have varying severity of illness which further complicates care regarding PAD. Some participants also reported the influence of response to therapy by pre-existing medical conditions or previous exposure to pharmacotherapy. The recognition of the need to individualise PAD management also contributes to frustrations as it precludes the use of uniform clinical PAD protocol.

\section{Preferences of healthcare providers}

Our participants reported that healthcare providers often have varying knowledge about PAD and individual preferences for specific medications, sedation levels, use of restraints, earplugs and care process. They also highlighted the problem of inconsistencies in PAD management provided by healthcare providers. Moreover, they reported that the choices of pharmacotherapy vary significantly among intensivists resulting in instances where patient's analgesics, sedatives or antipsychotics were changed drastically without proper patient assessment when there was a change-over of intensivist. Participants were also frustrated by inconsistent PAD management approaches among nurses and they recognised a difference in PAD management between day shifts and night shifts. They felt that night nurses tend to sedate patients more deeply compared with day nurses. These perceptions exist despite the fact that most nurses work both day and night shifts. They felt that these care differences impacted on how patients were managed over the duration of their ICU stay and potentially may contribute to prolonged ICU length of stay. Some participants reported that the variability of PAD care provided by healthcare professionals could negatively impact patient care and it became a constant frustration of bedside nurses.

\section{Theme: the factors impacting PAD management}

The third main theme that emerged from the data centred on factors that impact PAD management. The participants reported that PAD management was complex and healthcare providers need to consider many factors when they cared for their patients from the perspective of PAD.

\section{Interprofessional dynamics}

The participants reported that healthcare team dynamics, communications between multidisciplinary healthcare teams and with the patients and family could positively or negatively influence patient care. For example, the participants felt that physicians should assess the patients and communicate with the bedside nurses before altering PAD pharmacotherapy. Bedside nurses often have a better understanding of the patients' clinical state compared with intensivists who often spend only a short time with the patients precluding them from recognising the trend of patients' clinical status. Moreover, participants felt it would be beneficial for the intensivists to explain the rationale of changing pharmacotherapy to the bedside 
nurses, therefore promoting education and understanding among healthcare professionals.

More importantly, the participants reported that it is important for the healthcare team to effectively communicate with patients and patients' family to promote their understanding, facilitate therapeutic rapport and build trust. It would also reduce patient and family anxiety. Moreover, effective communication with patients and family could provide clues to appropriate and individualised non-pharmacological PAD therapy.

\section{Environmental factors}

The participants reported many aspects of the environment that they felt could impact the patient well-being. These included overhead announcements, monitors, patient positioning in the room, bright lighting and constant healthcare professional interaction with the patients. As noted by multiple participants, there were a variety of distractions and disruptions to the patient that were felt to negatively impact PAD. Participants suggested ways to minimise the negative environmental effects on patient well-being, such as clustering medical procedures, turning lights off at night and minimising overhead announcements in patient rooms.

\section{Family input}

The participants reported that family members could be helpful in the care processes or may be disruptive to the patient and healthcare teams that could be stress inducing for the patients. This relates to interactions that healthcare providers had with the patients and/or how the family engaged with the patient. The participants also felt that healthcare providers could better incorporate family members into care through education on how best to interact with the patients, while also involving family members to further understand patient's baseline conditions and care preferences.

\section{DISCUSSION}

Nurses play a unique and integral role in the management of PAD in the ICU. ${ }^{40}$ Therefore, understanding the nurses' beliefs, perceptions and experiences of PAD management is imperative in the development of practice improvements. Qualitative studies have been conducted to explore perceptions of and barriers to the management of pain ${ }^{41}$ and delirium ${ }^{28}{ }^{42-44}$ separately. However, to the best of authors' knowledge, this is one of few qualitative studies that described the management of PAD as a whole. In this study, we identified three themes: (1) the professional perspectives on patient wakefulness state, (2) the professional perspectives on PAD management of critically ill patients and (3) the factors impacting PAD management.

Our data demonstrated that there were divergent opinions on the optimal sedation level of critically ill patients despite the $2018 \mathrm{PAD}$ guidelines ${ }^{45}$ recommending a light sedation level in critically ill mechanically ventilated patients. The rationale behind the participants' preference for deep sedation is the fear of psychological trauma or PTSD as a consequence of awareness of ICU surroundings. This is contrary to the evidence suggesting that light sedation is not associated with PTSD. ${ }^{46}$ Indeed, studies have shown that more than $60 \%$ of critically ill mechanically ventilated patients are deeply sedated. ${ }^{47}$ The participants' preferences of deep sedation and the published observed care gaps are potentially secondary to a knowledge gap surrounding the benefits of light sedation in reducing ventilation duration and ICU length of stay. ${ }^{12}$ Since education interventions have been shown to improve the quality of sedation and analgesia of critically ill patients, ${ }^{49}$ a nurse-focused education intervention may potentially change nurses' beliefs and close the care gap.

In regard to the professional perspectives on PAD management, the participants were overall dissatisfied with their lack of understanding of the complexity of PAD management, the inconsistency of care plan and the suboptimal communications among healthcare providers. The participants appeared to have a good understanding that patients' variable responses to analgesics, sedatives and antipsychotics are related to patients' underlying comorbidities, severity of illness and drug metabolism. However, participants preferred to have further education surrounding these topics. This further supports the implementation of an educational intervention. The inconsistency of PAD management identified in this study is not unique to our centre. ${ }^{28}$ From the perspectives of suboptimal communications among healthcare providers, it has been recognised as a common phenomenon and studies have demonstrated strategies to improve communications between nurses and physicians. ${ }^{50}$ This also highlights the importance of a good nurse-physician relationship in the work environment to provide good quality of care. ${ }^{33}$

Through exploring nurses' beliefs, perceptions and experiences of PAD management, the participants have raised a few potential facilitators of PAD management. Participants have suggested that effective communications between the healthcare teams and patients must be established in order to prevent patient confusion. Educating family members on PAD was also identified as another facilitator. In addition, the participants discussed the important roles that patients' family members play in their overall well-being which should be considered in a $\mathrm{PAD}$ management strategy. These ideas are integral to the development of a multifaceted and multidisciplinary PAD quality improvement programme.

Overall, the themes emerged from this study have significant implications in the planning and the delivery of quality improvement interventions to improve the implementation of the PAD guidelines. It is apparent that interventions should target the improvement of (1) nurses' knowledge, (2) communications among healthcare providers and patients/family and (3) patients' family members' understanding of PAD. Finally, it was discovered that many nurses were interested in participating in quality improvement initiatives and they believed that 
having nurses contribute to the development of various quality improvement interventions would be the best approach. This insight directly influences our approach in developing a PAD quality improvement programme by changing it from a 'top down' approach to a 'bottom up' approach. By conducting nurse focus groups to listen to the nurses' voices, we have provided them with some control over their behaviour in managing PAD in the ICU. Moreover, by engaging the nurses in open discussions on the topic of PAD management, we were able to elicit potential facilitators of PAD guideline implementation in order to allow us to design subsequent quality improvement interventions.

The strengths of this study include the examination of nurses' attitudes towards PAD management in a Canadian community ICU. Six analysts from a diverse background performed data analysis (three generated codes and three developed themes) to allow researcher triangulation and to ensure validity of results. The use of focus group as a method of data collection facilitated the emergence of personal and group beliefs, perceptions and opinions. The limitations of this study include the lack of audio recording for transcription. However, there were dedicated notetakers to transcribe detailed notes including direct quotes to allow for data analysis. Moreover, this is a single-centre study which might affect generalisability. However, we have provided details of the setting and context. Moreover, the themes that emerged from this study are likely not unique to our centre. Therefore, local and international readers can interpret the study results with adequate setting and contextual information and apply the ideas to their centres.

In summary, this qualitative study provided unique nurses' perspectives on PAD management as it was currently understood in the ICU. It also provided insight to the further development of quality improvement interventions aiming to improve $\mathrm{PAD}$ management in the ICU. The results of this study have informed the development of subsequent multidisciplinary quality improvement interventions. More importantly, the process of conducting these focus groups engaged the nurses in the upcoming quality improvement programme as many nurses provided invaluable ideas and volunteered to participate in these initiatives.

\section{CONCLUSIONS}

ICU nurses have differing perspectives on optimal PAD management. The participants in this study provided various improvement strategies for the optimisation of PAD management which will be important to consider in future practice changes to enhance PAD management. Engaging the ICU nurses in focus groups has increased nurse understanding and involvement in this topic which in turn may improve the success and sustainability of implementing quality improvement interventions.
Acknowledgements We would like to acknowledge all the critical care nurses who attended the focus group sessions and Elayn Young, our critical care manager who has been very supportive of this initiative.

Contributors JLYT planned the study, developed and conducted the focus groups, analysed the data and prepared the manuscript. KR planned the study, developed and conducted the focus groups and analysed the data. FM and RM analysed the data. PY and CM collected the data. MC collected and analysed the data. DF planned the study, conducted the focus groups and analysed the data. ML planned the study, developed and conducted the focus groups, analysed the data and prepared the manuscript.

Funding This work was supported by the McMaster University, Department of Medicine, E.J. Moran Campbell Internal Research Career Award 2015-2018 (Jennifer LY Tsang).

Competing interests None declared.

Patient consent for publication Not required.

Ethics approval This study was approved by institutional research ethics board in May 2016 (code: 2016-05-001).

Provenance and peer review Not commissioned; externally peer reviewed.

Data sharing statement Data will be available if requested.

Open access This is an open access article distributed in accordance with the Creative Commons Attribution Non Commercial (CC BY-NC 4.0) license, which permits others to distribute, remix, adapt, build upon this work non-commercially, and license their derivative works on different terms, provided the original work is properly cited, appropriate credit is given, any changes made indicated, and the use is non-commercial. See: http://creativecommons.org/licenses/by-nc/4.0/.

\section{REFERENCES}

1. Mansouri $P$, Javadpour S, Zand F, et al. Implementation of a protocol for integrated management of pain, agitation, and delirium can improve clinical outcomes in the intensive care unit: a randomized clinical trial. J Crit Care 2013;28:918-22.

2. Ely EW, Shintani A, Truman B, et al. Delirium as a predictor of mortality in mechanically ventilated patients in the intensive care unit. JAMA 2004;291:291.

3. Ouimet S, Kavanagh BP, Gottfried SB, et al. Incidence, risk factors and consequences of ICU delirium. Intensive Care Med 2007;33:66-73.

4. Girard TD, Pandharipande PP, Ely EW. Delirium in the intensive care unit. Critical Care 2008;12:S3-9.

5. Ely EW, Gautam S, Margolin R, et al. The impact of delirium in the intensive care unit on hospital length of stay. Intensive Care Med 2001;27:1892-900.

6. Dubois MJ, Bergeron N, Dumont M, et al. Delirium in an intensive care unit: a study of risk factors. Intensive Care Med 2001;27:1297-304.

7. Milbrandt EB, Deppen S, Harrison PL, et al. Costs associated with delirium in mechanically ventilated patients. Crit Care Med 2004;32:955-62.

8. McAvay GJ, Van Ness PH, Bogardus ST, et al. Older adults discharged from the hospital with delirium: 1-year outcomes. J Am Geriatr Soc 2006;54:1245-50.

9. Girard TD, Jackson JC, Pandharipande PP, et al. Delirium as a predictor of long-term cognitive impairment in survivors of critical illness. Crit Care Med 2010;38:1513-20.

10. Salam A, Tilluckdharry L, Amoateng-Adjepong $Y$, et al. Neurologic status, cough, secretions and extubation outcomes. Intensive Care Med 2004;30:1334-9.

11. Lin SM, Liu CY, Wang CH, Shu-Min L, Chien-Ying L, Han-Pin K, et al. The impact of delirium on the survival of mechanically ventilated patients. Crit Care Med 2004;32:2254-9.

12. Barr J, Fraser GL, Puntillo K, et al. Clinical practice guidelines for the management of pain, agitation, and delirium in adult patients in the intensive care unit. Crit Care Med 2013;41:263-306.

13. Skrobik Y, Ahern S, Leblanc M, et al. Protocolized intensive care unit management of analgesia, sedation, and delirium improves analgesia and subsyndromal delirium rates. Anesth Analg 2010;111:451-63.

14. Girard TD, Kress JP, Fuchs BD, et al. Efficacy and safety of a paired sedation and ventilator weaning protocol for mechanically ventilated patients in intensive care (Awakening and Breathing Controlled trial): a randomised controlled trial. Lancet 2008;371:126-34.

15. Vasilevskis EE, Ely EW, Speroff T, et al. Reducing iatrogenic risks: ICU-acquired delirium and weakness-crossing the quality chasm. Chest 2010;138:1224-33. 
16. McKee G, Kerins M, Hamilton G, et al. Barriers to ESC guideline implementation: results of a survey from the European Council on Cardiovascular Nursing and Allied Professions (CCNAP). Eur J Cardiovasc Nurs 2017;16:678-86.

17. Brotons C, Lobos JM, Royo-Bordonada MÁ, et al. Implementation of Spanish adaptation of the European guidelines on cardiovascular disease prevention in primary care. BMC Fam Pract 2013;14:36-42.

18. Abrahamson KA, Fox RL, Doebbeling BN. Facilitators and barriers to clinical practice guideline use among nurses. Am J Nurs 2012;112:26-36.

19. Jun J, Kovner CT, Stimpfel AW. Barriers and facilitators of nurses' use of clinical practice guidelines: An integrative review. Int J Nurs Stud 2016;60:54-68.

20. Ploeg J, Davies B, Edwards N, et al. Factors influencing bestpractice guideline implementation: lessons learned from administrators, nursing staff, and project leaders. Worldviews Evid Based Nurs 2007;4:210-9.

21. Ferrante D, Konfino J, Linetzky B, et al. Barriers to prevention of cardiovascular disease in primary care settings in Argentina. Rev Panam Salud Publica 2013;33:259-66.

22. Hobbs FD, Erhardt L. Acceptance of guideline recommendations and perceived implementation of coronary heart disease prevention among primary care physicians in five European countries: the Reassessing European Attitudes about Cardiovascular Treatment (REACT) survey. Fam Pract 2002;19:596-604.

23. Erhardt L, Komajda M, Hobbs FD, et al. Cardiologists' awareness and perceptions of guidelines for chronic heart failure. The ADDress your Heart survey. Eur J Heart Fail 2008;10:1020-5.

24. Hadely KA, Power E, O'Halloran R. Speech pathologists' experiences with stroke clinical practice guidelines and the barriers and facilitators influencing their use: a national descriptive study. BMC Health Serv Res 2014;14:1-41.

25. Damschroder LJ, Aron DC, Keith RE, et al. Fostering implementation of health services research findings into practice: a consolidated framework for advancing implementation science. Implement Sci 2009;4:50.

26. Kitson AL, Rycroft-Malone J, Harvey G, et al. Evaluating the successful implementation of evidence into practice using the PARiHS framework: theoretical and practical challenges. Implement Sci 2008;3:1-12.

27. Devlin JW, Fong JJ, Howard EP, et al. Assessment of the delirium in the intensive care unit: nursing practices and preceptions. Crit Care Med 2008;15:555-65.

28. Palacios-Ceña D, Cachón-Pérez JM, Martínez-Piedrola R, et al. How do doctors and nurses manage delirium in intensive care units? A qualitative study using focus groups. BMJ Open 2016;6:e009678-11.

29. O'Brien BC, Harris IB, Beckman TJ, et al. Standards for reporting qualitative research: a synthesis of recommendations. Acad Med 2014;89:1245-51.

30. Neergaard MA, Olesen F, Andersen RS, et al. Qualitative description - the poor cousin of health research? BMC Med Res Methodol 2009:9:52-6.

31. Sandelowski M. What's in a name? Qualitative description revisited. Res Nurs Health 2010;33:77-84.

32. Parsons M, Greenwood J. A guide to the use of focus groups in health care research: Part 1. Contemp Nurse 2000;9:169-80.
33. Kitzinger J. Qualitative Research: Introducing focus group. BMJ Open 1995:299-302.

34. Jones S, Torres V, Armanio J. Negotiating the Complexities of Qualitative Research in Higher Education: Fundamental Elements and Issues. Second Edition. New YorkRoutledge: NY, 2014.

35. Adom D, Yeboah A, Ankrah AK. Constructivism philosophical paradigm: implication for research, teaching and learning. GJAHSS 2016;4:1-9.

36. Palinkas LA, Horwitz SM, Green CA, et al. Purposeful sampling for qualitative data collection and analysis in mixed method implementation research. Adm Policy Ment Health 2015;42:533-44.

37. Guest G, Namey EE, Mitchell ML. Collecting qualitative data: a field manual for applied research. Thousands Oaks: Sage Publication 2013.

38. Maximous R, Miller F, Tan C, et al. Pain, agitation and delirium assessment and management in a community medical-surgical ICU: results from a prospective observational study and nurse survey. BMJ Open Qual 2018;7:e000413.

39. Noble H, Smith J. Issues of validity and reliability in qualitative research. Evid Based Nurs 2015;18:34-5.

40. Balas MC, Vasilevskis EE, Burke WJ, et al. Critical care nurses' role in implementing the "ABCDE bundle" into practice. Crit Care Nurse 2012;32:35-47.

41. Hetland B, Guttormson J, Tracy MF, et al. "Sedation is tricky": A qualitative content analysis of nurses' perceptions of sedation administration in mechanically ventilated intensive care unit patients. Aust Crit Care 2018;31:153-8.

42. Yevchak A, Steis M, Diehl T, Fick D, et al. Managing delirium in the acute care setting: a pilot focus group study. Int J Older People Nurs 2012; 7:152-62.

43. Yue P, Wang L, Liu C, et al. A qualitative study on experience of nurses caring for patients with delirium in ICUs in China: Barriers, burdens and decision making dilemmas. Int J Nurs Sci 2015;2:2-8.

44. van de Steeg $L$, Langelaan $M$, ljkema $R$, et al. Improving delirium care for hospitalized older patients. A qualitative study identifying barriers to guideline adherence. J Eval Clin Pract 2014;20:813-9.

45. Devlin JW, Skrobik Y, Gélinas C, et al. Clinical practice guidelines for the prevention and management of pain, agitation/sedation, delirium, immobility, and sleep disruption in adult patients in the ICU. Crit Care Med 2018;46:e825-e873.

46. Rock LF. Sedation and its association with posttraumatic stress disorder after intensive care. Crit Care Nurse 2014:34:30-7.

47. Shehabi Y, Chan L, Kadiman S, et al. Sedation depth and longterm mortality in mechanically ventilated critically ill adults: a prospective longitudinal multicentre cohort study. Intensive Care Med 2013;39:910-8.

48. Hodgson C, Bellomo R, Berney S, et al. Early mobilization and recovery in mechanically ventilated patients in the ICU: a bi-national, multi-centre, prospective cohort study. Crit Care 2015;19:19-81.

49. Walsh TS, Kydonaki K, Antonelli J, et al. Staff education, regular sedation and analgesia quality feedback, and a sedation monitoring technology for improving sedation and analgesia quality for critically ill, mechanically ventilated patients: a cluster randomised trial. Lancet Respir Med 2016;4:807-17.

50. Kvande M, Lykkeslet E, Storli SL. ICU nurses and physicians dialogue regarding patients clinical status and care options-a focus group study. Int J Qual Stud Health Well-being 2017;12:1267346-8. 